\title{
A Familial Case with Holt-Oram Syndrome with a Novel TBX5 Mutation
}

\author{
Beom Hee Lee ${ }^{1,2}$, Yoo-Mi Kim', Gu-Hwan Kim², Young-Hwue Kim ${ }^{1}$ and Han-Wook Yoo ${ }^{1,2 *}$ \\ Department of Pediatrics', Medical Genetics Center², Asan Medical Center children`s Hospital, University of Ulsan College of Medicine, Seoul, Korea
}

Holt-Oram syndrome (HOS) is the most common heart-hand syndrome, which is inherited in an autosomal dominant manner, but most cases are sporadic. This condition is characterized by upper-extremity malformations involving radial-ray, thenar, and carpal bones, and congenital heart malformations including atrial septal defect and ventricular septal defect. It is caused by mutations in the TBX5 gene. In this report, a Korean case with HOS is described, which is inherited from her father. A novel nonsense mutation, p.Glu294*, was identified. This is the first Korean case with HOS confirmed by genetic testing.

Key words: Holt-Oram syndrome, TBX5, Mutation

\section{Introduction}

Holt-Oram syndrome (HOS; OMIM 142900) is a congenital disorder, inherited in an autosomal dominant manner. HOS primarily affects heart and hand, which is characterized by upper-extremity malformations involving radial-ray, thenar, and carpal bones and congenital heart malformations including atrial septal defect and ventricular septal defect. ${ }^{1,2)}$ Some patients have cardiac conduction defect. However, clinical manifestations of HOS have been variable among patients. ${ }^{2)} \mathrm{HOS}$ is caused by mutations in the TBX 5 gene. ${ }^{3)}$

Although HOS is the most common heart-hand syndrome with its estimated prevalence of 1 over 100,000 live births,") only a few Korean cases have been reported. ${ }^{4-6)}$ Moreover, no case has been reported in Korean population confirmed by genetic testing. In this report, a familial case with HOS is described with novel TBX5 mutation. This is the first Korean case with HOS confirmed by genetic testing.

\section{Case Report}

The patient was the first off-spring of non-consanguineous Korean parents. She was born after 40 weeks of gestation. Her prenatal evaluation had been uneventful. Postnatal evaluation was also normal except left hand anomaly. Left thumb was triphalangeal and hypoplastic, and the other extremities were normal (Fig. 1). At 1 month of age, respiratory difficulty was noted with tachypnea, and systolic murmur was audible at left sterna border. Echocardiography revealed large secundum atrial septal defect with left-to-right shunt. In addition, perimembranous inlet-trabecular type of ventricular septal defect was found with septal aneurysm. At 2 months of age, patch closure for ventricular septal defect and atrial septal defect was done. After the operation, the diaphragm plication was performed for right diaphragmatic palsy. Of note, her father had been also given heart operation for ventricular septal defect at 10 years of age, and both

Received: 17 Nov 2012, Revised: 11 Dec 2012, Accepted: 18 Dec 2012, Published: 31 Dec 2012

*Corresponding author: Han-WookYoo, M.D., Ph.D.

Genome Research Center for Birth Defects and Genetic Diseases, Department of Pediatrics, Asan Medical Center, University of Ulsan College of Medicine, 388-1 Pungnap-Dong, Songpa-Gu, Seoul 138-736, Korea.

Tel: +82-2-3010-3374, Fax: +82-2-473-3725, E-mail: hwyoo@amc.seoul.kr

*Conflicts of interest: The authors have nothing to disclose or conflicts of interest

(c) This is an open-access article distributed under the terms of the Creative Commons Attribution Non-Commercial License (http://creativecommons.org/licenses/by-nc/3.0/) which permits unrestricted non-commercial use, distribution, and reproduction in any medium, provided the original work is properly cited.

(c) Copyright 2012 by the Korean Society of Medical Genetics 


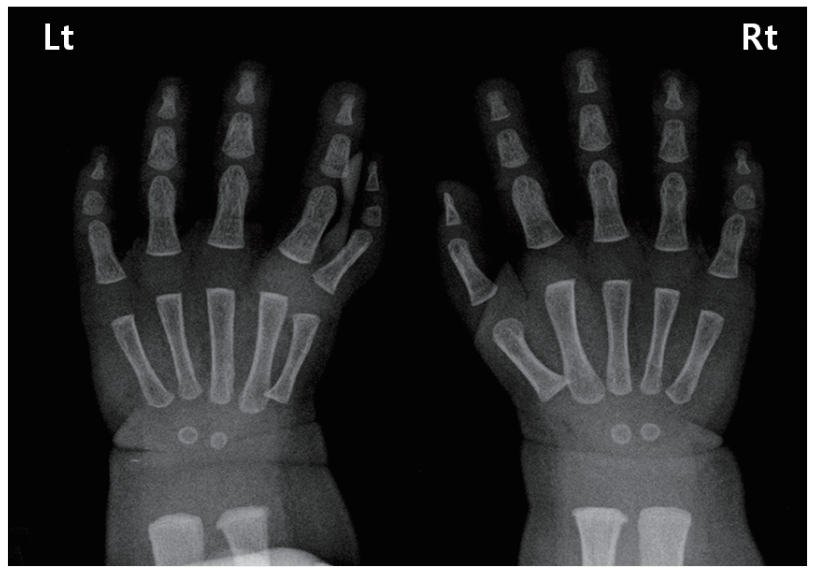

Fig. 1. Left triphalangeal thumb and normal right thumb.

3T CCCAATACCA GT GT GAGA ATGGT GT TTCC GGC Normal

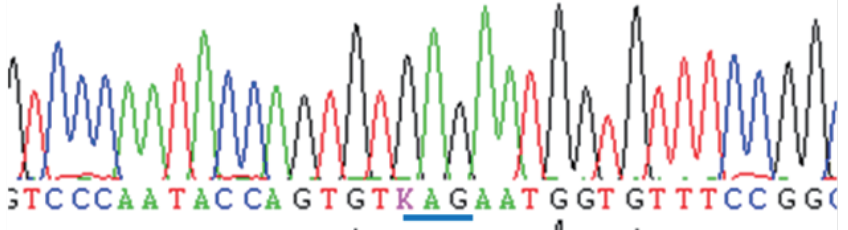

Patient

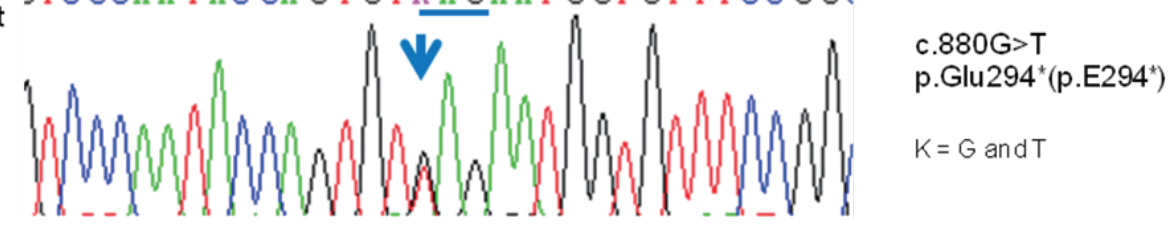

Fig. 2. Partial sequence of the $T B X 5$ gene. The patient and her father carries a novel nonsense mutation, c.880G $>$ T (p.Glu294*).

his thumbs were abnormal. Under the impression of familial HOS, genetic testing for the TBX5 gene was done, using genomic DNA from peripheral leukocytes. The patient and her father shared the novel nonsense mutation, c.880G>T (p.Glu294*) (Fig. 2). Thumb reconstruction operation was performed at two years of age. Until 5 years of age, she showed normal development with normal growth profiles; her height was $100 \mathrm{~cm}\left(10^{\text {th }}\right.$ percentile) and weight was $14.2 \mathrm{~kg}$ ( $25^{\text {th }}$ percentile). Holter monitoring has been performed annually, which revealed intermittent sinus bradycardia, sinus node dysfunction with junctional escape beat, but no episode of atriventricular block wasseen.

\section{Discussion}

Upper limb anomalies in HOS can be variable among patients. The deformities may be unilateral or bilateral, and symmetric or asymmetric. These are often more severe in left side than in right side. Hand deformities range from triphalangeal, as in our patient, or absent thumb to phocomelia. In some patients, radial aplasia or hypoplasia can be also noted. ${ }^{1,2)}$ Although most cases with HOS have upper limb anomalies, cardiac defects can be seen in 75\% of the patients. The most common cardiac anomalies are atrial septal defect and ventricular septal defect, as in our case, but some patients have complex cardiac defects including conotruncal malformations. ${ }^{7)}$ The long-term prognosis of a patient with HOS usually depends on the severity of cardiac defects. Especially patients can have cardiac conduction disease, which starts as sinus bradycardia, but it can progress to high-degree atrioventricular block with or without atrial fibrillation. ${ }^{5}$

In the diagnostic process, the following diseases should be differentiated from HOS. SALL4-related disorders include radial ray malformations like $\mathrm{HOS}$, Indeed, in rare cases with HOSfeature, SALL4 mutations are found. However, ocular and renal abnormalities are exclusively involved in SALL4-related disorders, but not in HOS. Townes-Brocks syndrome is another disease with similar phenotype to that of HOS, but it also includes renal-ear- analradial anomalies, is caused by mutations in $S A L L 1 .{ }^{8}$ Other diseases to be excluded are heart-hand syndrome II/III, Fanconi anemia, VACTERL sequence, and teratogen exposure like thalidomide 
and valproate. The important feature to differentiate HOS from these similar conditions is the absence of ulnar ray malformation without radial ray, anomalies including kidney, eye, vertebrae, ear, and anus in $\mathrm{HOS}^{2}{ }^{2}$

TBX5 is a member of the T-box family of transcription factors, and the T-box domain is the DNA-binding site. TBX5 plays an important role in cardiac and limb developments.) TBX5 mutations are found in over $70 \%$ of patients with HOS. Currently, about 90 TBX5 mutations have been identified, most of which are private and point mutations (http://www.hgmd.org/). The novel nonsense mutation found in this case is expected to lead to premature truncated protein, which may be degraded and fail to be localized in the nucleus, where TBX5 could have played its proper role.

Most cases with HOS are sporadic cases, but our case is a familial case with paternal inheritance. More cases with HOS should be identified to characterize the clinical and genetic features of Korean patients with HOS.

\section{Acknowledgements}

We thank our patient and her family members for their participation in this study. This study was supported by Grant A080588 from the Korean Ministry of Health, Welfare and Family Affairs.

\section{References}

1. Holt M, Oram S. Familial heart disease with skeletal malformations. $\mathrm{Br}$ Heart J 1960;22:236-42.

2. McDermott DA, Bressan MC, He J, Lee JS, Aftimos S, Brueckner M, et al. TBX5 genetic testing validates strict clinical criteria for Holt-Oram syndrome. Pediatr Res 2005;58:981-6.

3. Basson CT, Bachinsky DR, Lin RC, Levi T, Elkins JA, Soults J, et al Mutations in human TBX5 [corrected] cause limb and cardiac malformation in Holt-Oram syndrome. Nat Genet 1997;15:30-5.

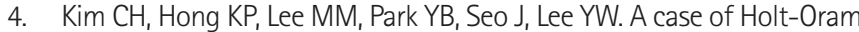
syndrome. Korean Circ J 1982;12:231-8.

5. Lee JG, Choi AN, Jee EG, Gwon TH, Lee YH, Lee SH, et al. A case of the Holt-Oram syndrome with unaffected parents diagnosed by antenatal ultrasonography. Korean J Obstet Gynecol 2000;43:2095-9.

6. Seo EJ, Kim GJ, Son MS, Lee ED. A case of the Hot-Oram syndrome which was prenatal diagnosed by ultrasonography in affected mother. Korean J Obstet Gynecol 2003;46:446-9.

7. Sletten $\sqcup$, Pierpont ME. Variation in severity of cardiac disease in HoltOram syndrome. Am J Med Genet 1996;65:128-32.

8. Kohlhase J. SALL1 mutations in townes-brocks syndrome and related disorders. Hum Mutat 2000;16:460-6. 